\title{
Análise temporal de combates de Muay-Thai de nível nacional: Efeitos da fase competitiva
}

\author{
Fabrício Boscolo DEL VECCHIO ${ }^{1 *}$, Jerônimo Jaspe Rodrigues SILVA
FARIAS$^{1}$
${ }^{1}$ Escola Superior de Educação Física, Universidade Federal de Pelotas (Brasil) \\ 2 Escola de Educação Física, Universidade Federal do Rio Grande do Sul (Brasil)
}

Recepción: 27/03/2015; Aceptación: 19/06/2015; Publicación: 26/06/2015.

\begin{abstract}
Resumo
Introdução: A análise temporal dos combates possibilita estudo dos padrões de luta nos esportes de combate, e se pode recriar os esforços em treinamentos específicos. Porém, não há informações disponíveis sobre análise temporal em diferentes níveis competitivos em lutas de Muay-Thai. Objetivo: Investigar as características temporais realizadas por atletas de Muay-Thai em competição oficial de nível nacional, segundo round, e comparar combates eliminatórios com disputas de medalhas. Materiais e métodos: Foram registradas 65 lutas masculinas de Campeonato Brasileiro. Empregouse estratégia de filmagem in loco e, para análise de tempo movimento, foi utilizado Software Kinovea ${ }^{\circ}$. Consideraram-se períodos de observação, preparação e interação, bem como relação de alta intensidade e baixa intensidade (AI:BI). A duração média dos rounds entre fases foi comparada com teste $t$ de Student. Aplicou-se modelo linear generalizado considerando a duração de esforço como variável dependente, e nível competitivo, round e tipo de esforço como independentes. 0 nível de significância foi de 5\%. Resultados: Em média, registraram-se de 7 a 8 s de observação, aproximadamente $3 \mathrm{~s}$ de preparação e 4 a 5,5 s de interação, com tempo de observação estatisticamente superior aos demais. Constatou-se diferença significante no tempo de interação entre lutas eliminatórias e finais (respectivamente 5,5 $\pm 0,3$ s e $3,7 \pm 0,5 \mathrm{~s} ; \mathrm{p}<0,05$ ). Ao se agruparem os tempos de observação e preparação como baixa intensidade (BI), e de interação como esforço de alta intensidade (AI), a relação AI:BI foi de 1:2 em lutas eliminatórias e de 1:3 nos combates finais. Conclusões: Conclui-se que o Muay-Thai se configura como uma modalidade de combate intermitente e que o tempo destinado à interação é inferior em confrontos finais quando comparados aos eliminatórios.
\end{abstract}

ORIGINAL PAPER

Palavras-chave: Artes marciais; desportos de combate; desempenho atlético; estudos de tempo e movimento.

\section{Temporal analysis of national level Muay-Thai matches: Effects of competitive phase}

\section{Abstract}

Introduction: Temporal analysis of matches allows the study of fighting patterns in combat sports. With this procedure, we can recreate the efforts in specific training. However, up to date have no information concerning temporal analysis considering competitive level in Muay-Thai. Objective: To investigate temporal characteristics of Muay-Thai athletes in official competition from national level, considering round, and to compare preliminary combats with those that had medal disputes. Material and methods: Were recorded 65 matches in a Brazilian championship. We conducted in loco recording. To time-motion analysis, Software Kinovea $^{\mathrm{TM}}$ was used. Were considered Observation, Preparation and Interaction periods, as well as high intensity and low intensity ratio (HI:LI). The average duration of the rounds between competitive phases (preliminary or finals) was compared with a $t$ test. A generalized linear model was applied considering effort duration as dependent variable and competitive level, round and effort type as

\section{Análisis temporal de combates de muay-thai de} nivel nacional: efectos de la fase de competición \section{Resumen}

Introducción: El análisis temporal de los enfrentamientos posibilita el estudio de los patrones de lucha en los deportes de combate. Mediante este procedimiento se pueden recrear los tipos de esfuerzo en entrenamientos específicos. Sin embargo, hasta la fecha no hay información sobre el muay-thai en relación al análisis temporal considerando la fase de competición. Objetivo: Investigar las características temporales de atletas de muay-thai en competición oficial de nivel nacional, considerando los asaltos, y comparar los combates preliminares con aquellos en los que hay disputa de medallas. Materiales y métodos: Se registraron 65 combates en categoría masculina del Campeonato de Brasil. Se utilizó una estrategia de filmación in loco y, para el análisis del tiempo de movimiento se utilizó el Software Kinovea ${ }^{\circledR}$. Se consideraron períodos de observación, preparación e interacción, así como la ratio alta intensidad y baja intensidad (AI:BI). La duración media de los asaltos entre las fases de competición (preliminares o finales) se comparó mediante el test $t$ de Student. Se aplicó un modelo linear generalizado considerando la duración del esfuerzo como variable dependiente y el nivel competitivo, asalto y tipo de esfuerzo como independientes. El

\footnotetext{
*E-mail: fabricio_boscolo@uol.com.br. Dirección: Rua Luiz de Camões, 625. Três Vendas, Pelotas, Rio Grande do Sul (Brasil). CEP: 96055-630.
} 
independent. Significant level was set in 5\%. Results: Were registered 7 to $8 \mathrm{~s}$ of Observation, near from $3 \mathrm{~s}$ of Preparation and 4 to $5.5 \mathrm{~s}$ of Interaction effort type, and the duration of Observation effort was significantly superior the others. Significant differences were observed in the Interaction between preliminary and finals (respectively $5.5 \pm 0.3 \mathrm{~s}$ and $3.7 \pm 0.5 \mathrm{~s} ; \mathrm{p}<0.05)$. Pooling the Observation and Preparation time as low intensity (LI), and Interaction as high intensity (HI) efforts, the HI:LI ratio was 1:2in eliminatory and 1:3 in the final matches. Conclusions: Muay-Thai is an intermittent combat sport, the duration of Interaction effort type is lower in final in comparison to preliminary matches.

Keywords: Martial arts; combat sports; athletic performance; time and motion studies. nivel de significación se fijó en el 5\%. Resultados: Como media, se registraron de 7 a $8 \mathrm{~s}$ de observación, aproximadamente $3 \mathrm{~s}$ de preparación y 4 a 5,5 s de interacción, y el tiempo de observación fue estadísticamente superior a los demás. Se observaron diferencias significativas en la interacción entre las fases preliminares y finales (respectivamente $5,5 \pm 0,3$ s y $3,7 \pm$ $0,5 \mathrm{~s} ; \mathrm{p}<0,05)$. Al agrupar los tiempos de observación y preparación como de baja intensidad (BI), y el interacción como esfuerzo de alta intensidad (AI), la relación AI:BI fue de 1:2 en las fases preliminares y 1:3 en los combates finales. Conclusiones: Se concluye que el muay-thai se configura como una modalidad de combate intermitente y que el tiempo destinado a la interacción es inferior en los combates finales comparación con los eliminatorios.

Palabras clave: Artes marciales; deportes de combate; rendimiento atlético; estudios de tiempo y movimiento.

\section{Introdução}

A análise de tempo e movimento (ATM) dos combates é um dos modos de se estudar os padrões de luta nas modalidades esportivas de combate (MEC) (Bridge, McNaughton, Close, \& Drust, 2013; Franchini \& Del Vecchio, 2011). Assim, reconhecem-se as demandas dos combates (Miarka, et al., 2012) e pode tentar mimetizar os esforços específicos em treinamentos para condicionamento dos atletas (Del Vecchio, Hirata, \& Franchini, 2011; Bridge, McNaughton, Close, \& Drust, 2013). Quanto à ATM, predominantemente, encontram-se estudos com judo (Miarka, et al., 2012), Brazilian jiu-jitsu (BJJ) (Andreato, et al., 2015), karate (Arriaza, 2009; Chaabène, et al., 2014), taekwondo (Heller, et al., 1998; Matsushigue, Hartmann, \& Franchini, 2009; Bridge, McNaughton, Close, \& Drust, 2013) e MMA (Del Vecchio, Hirata, \& Franchini, 2011).

De origem tailandesa, o Muay-Thai se caracteriza como luta de percussão em função de suas regras de ação, e seus gestos específicos são realizados com membros superiores (socos e cotoveladas) e inferiores, com chutes e joelhadas (Turner, 2009). Do ponto de vista competitivo, a luta se dá em combates compostos por três a quatro rounds de três minutos com um minuto de intervalo entre eles, conforme estabelecido pela Federação Internacional de Muay-Thai Amador (IFMA). Fisiologicamente, em um combate de Muay-Thai podem ser atingidos valores de frequência cardíaca de $183 \mathrm{bpm}$ e concentração de lactato de $12,55 \mathrm{mmol} / \mathrm{L}$, e quanto à ATM, 40\% do tempo total é destinado à fase de estudo, 20,4\% a ataques em situações de clinches e 39,6\% a ataques à distância (Cappai, et al., 2012). Em combates simulados, observou-se consumo máximo de oxigênio $\left(\mathrm{VO}_{2 \max }\right)$ de aproximadamente $48,5 \mathrm{~mL} \cdot \mathrm{min}^{-1} \cdot \mathrm{kg}^{-1}$, sendo que o valor máximo foi atingido durante o segundo round, e valores médios de frequência cardíaca de 180 bpm (Crisafulli, et al., 2009). Ainda quanto à ATM, estudo prévio com lutas de nível amador registrou períodos de observação, preparação e interação de $5 \mathrm{~s}, 2 \mathrm{~s}$ e $5 \mathrm{~s}$, respectivamente em combates envolvendo competidores uruguaios (Silva, Del Vecchio, Picanço, Takito, \& Franchini, 2011). Neste sentido, o emprego da ATM na prescrição de exercícios especiais ainda é inicial.

Empiricamente, sugere-se que técnicos de Muay-Thai usem $5 \mathrm{~s}$ de esforço por $5 \mathrm{~s}$ de pausa como modo de condicionamento físico de lutadores (Turner, 2009). De modo complementar, estudo recente empregou treinamento intermitente de alta intensidade por dez dias, duas vezes ao dia para atletas de Muay-Thai, com esforços entre 6 e 60 s (intensidade entre $80 \%$ e $100 \%$ do $\mathrm{VO}_{2 \max }$ ), com pausas entre 18 e $120 \mathrm{~s}$, e constatou alterações no perfil de estresse oxidativo dos competidores (Ugras, 2013). No entanto, não há evidências científicas que suportem tais demandas temporais, caso o uso de gestos específicos sejam usados para o condicionamento físico dos competidores.

Ademais, pouco se sabe como um evento competitivo pode modificar o padrão de combate no Muay-Thai em lutas sucessivas. Até o momento, reconhece-se que combates sucessivos podem induzir à fadiga, diminuição do desempenho físico em testes motores e elevar o estresse metabólico (Fry, Schilling, Fleck, \& Kraemer, 2011; Andreato, et al., 2015; Barbas, et al., 2011), mas não há informações sobre seu efeito na ATM, o que poderia gerar impacto direto na tática de treinamento e 
estratégia competitiva.Com base nisso, o objetivo do presente estudo foi investigar e comparar a duração de diferentes tipos de esforços físicos em lutas eliminatórias e finais de Muay-Thai em competição oficial de nível nacional.

\section{Materiais e métodos}

0 estudo se caracteriza como observacional transversal, foi realizado com consentimento da Confederação Brasileira de Muay-Thai Tradicional, detentora dos direitos sobre a organização e divulgação midiática do evento, e foi aprovado previamente pelo comitê local de ética em pesquisa com seres humanos (Protocolo número 21623313.4.0000.5313). A fase competitiva - eliminatória (referente às lutas antecedentes às finais) ou final (disputa por primeiro e segundo lugar), foi considerada como principal variável independente, seguida por round e tipo de esforço.

A população foi composta por atletas do sexo masculino, praticantes de Muay-Thai, com dezesseis anos de idade ou mais, alocados nas seleções estaduais representadas no Campeonato Brasileiro de Muay-Thai de 2011. Foram gravadas todas as 65 lutas realizadas em evento chancelado pela Confederação Brasileira de Muay-Thai Tradicional (CBMTT) nos dias 30/04 e 1/05 de 2011, no qual treze estados brasileiros se encontravam devidamente representados por suas respectivas seleções.

Os combates foram registrados in loco e em vídeo para posterior análise e interpretação das variáveis descritas a seguir, considerando cada um dos lutadores de cada combate. Para a filmagem, foi utilizada câmera Sony ${ }^{\mathrm{TM}}$, modelo DS40, fixada em tripé com $180 \mathrm{~cm}$ de altura. Os vídeos foram armazenados em cartões de memória SD de 8GB e 4GB, bem como em computador portátil (Lenovo G550, Pentium $®$ Dual-Core CPU T4300 @ 2.10GHz - 2,00GB RAM - Windows 7 Home Basic 32 Bits - Copyright@ 2009 Microsoft Corporation). Cada luta foi numerada por fase competitiva, e cada competidor recebeu uma letra identificadora. 0 software Kinovea ${ }^{\text {TM }}$ foi empregado para a visualização de cada combate, como descrito previamente (Silva, Del Vecchio, Picanço, Takito, \& Franchini 2011). Com o respectivo software, as lutas foram exibidas em câmera lenta $(50 \%$ da velocidade normal de $60 \mathrm{fps}$ ), quando ocorriam as identificações e classificações dos blocos de esforço, explicitados a seguir. Os scouts, com registros sequenciais dos tipos de esforço, por luta e round, foram realizados em planilhas específicas do software Microsoft Excel ${ }^{\mathrm{TM}} 2010$ (Del Vecchio, Hirata, \& Franchini, 2011).

De acordo com as regras do evento, as lutas eliminatórias podiam chegar a até três rounds, e as finais poderiam chegar a quatro rounds. Para minimizar os problemas relacionados à variação entre avaliadores, apenas um sujeito foi treinado previamente por 34 horas e, então, ficou responsável por todas as análises. Buscou-se minimizar a chance de erro ao se considerarem exclusivamente a temporalidade, a partir de três níveis de esforços, e não tipos ou direções das técnicas aplicadas, dado que estudo prévio detectou limitações na validação desta última variável (Miarka, et al., 2011). Embora não se tenha testado a objetividade e reprodutibilidade no presente estudo, investigação prévia com Muay-Thay e Mixed Martial Arts apresentaram valores elevados, com coeficiente de correlação intraclasse superior a 0,98 (Silva, Del Vecchio, Picanço, Takito, \& Franchini 2011; Del Vecchio, Hirata, \& Franchini, 2011). Pesquisa prévia com karatê apresentou valores de coeficiente de correlação intraclasse superiores a 0,9 para atividades preparatórias, tempo de luta e de pausa, exibindo alta reprodutibilidade da ATM (Chaabène, et al., 2014).

Foram registradas as seguintes variáveis:

(a) Tempo de luta por round: para essa variável, o critério de identificação do tempo de combate o período entre a voz de comando do árbitro para iniciar o combate, a voz de comando para parar o combate e a voz de comando para terminar a luta (Silva, Del Vecchio, Picanço, Takito, \& Franchini 2011).

(b) Tempo de observação: compreende períodos de estudos do adversário (Cappai, et al., 2012), com predomínio de deslocamentos em baixa intensidade no ringue, sem contato entre os competidores (Buse, 2009; Arriaza, 2009). 
(c) Tempo de preparação: compreende troca de golpes em baixa intensidade, ataques para mensuração da distância e aplicações técnicas isoladas (Buse, 2009; Arriaza, 2009).

(d) Tempo de interação: engloba períodos de alta intensidade, nos quais há intensidade elevada nas trocas de golpes, e nos quais geralmente ocorrem as situações determinantes do êxito competitivo, como pontos e knock-outs (Buse, 2009; Arriaza, 2009).

Para análise estatística dos dados, após verificação da normalidade em sua distribuição observada pelo teste de Shapiro-Wilk, os mesmos são apresentados com média, erro padrão (ep) e intervalo de confiança de 95\%. Para estatística inferencial, inicialmente a duração dos rounds entre fases foi comparada com teste $t$ de Student para amostras independentes.

Foi empregado modelo linear generalizado, a partir de regressão linear simples, assumindose a duração de esforço como variável dependente. Fase competitiva, round e tipo de esforço (observação, preparação e interação) foram consideradas como variáveis independentes. Quando observadas diferenças significantes, as mesmas foram localizadas com teste $t$ de Student como posthoc e ajustamento de Bonferroni para medidas repetidas. 0 nível de significância foi estabelecido em $\mathrm{p}<0,05$, e são apresentados os valores do poder das análises multivariadas.

\section{Resultados}

Ocorreram 47 lutas eliminatórias, das quais foram analisados 123 rounds em que se realizaram 2863 blocos de esforços, os quais foram organizados como observação, preparação e interação. Após estas lutas eliminatórias foram realizadas 18 lutas finais, das quais derivaram 53 rounds e 1412 blocos de esforços.

Das lutas eliminatórias, 12 (25\%) não terminaram ao final do terceiro round. 0 tempo de luta por round foi de $119 \pm 26 \mathrm{~s}$, com $119,7 \pm 18,5 \mathrm{~s}$ no primeiro round, $125,1 \pm 45,7 \mathrm{~s}$ no segundo round, $119,7 \pm 22,8 \mathrm{~s}$ no terceiro round. Quanto às finais, 12 lutas $(66 \%)$ terminaram antes do último round, e se registrou como tempo de luta por round $122 \pm 25 \mathrm{~s}$, com $132,1 \pm 33$, $9 \mathrm{~s}$ no primeiro round, $118,3 \pm 44,0$ s no segundo, $117,7 \pm 20,6$ no terceiro e $123,0 \pm 2,0$ s no quarto round. Destaca-se que não houve diferenças significantes na duração total dos rounds de acordo com a fase competitiva $(t=-0,32 ; p=0,74)$.

$\mathrm{Na}$ análise multivariada, o teste de igualdade de variância de Levene apresentou $\mathrm{F}_{20,4254}=$ 26,33, com $p<0,001$. Observaram-se diferenças significantes nas durações dos esforços para fase competitiva $\left(\mathrm{F}_{20,1}=4,5 ; p=0,01\right.$; poder $\left.=0,52\right)$, round $\left(\mathrm{F}_{20,3}=4,88 ; p=0,002\right.$; poder $\left.=0,91\right)$, e tipo de esforço $\left(\mathrm{F}_{20,2}=82,59 ; p<0,001\right.$; poder $\left.=1,0\right)$, com interações significantes entre categoria e round $\left(\mathrm{F}_{20,2}=3,95 ; p=0,02\right.$; poder $\left.=0,71\right)$ e entre categoria e tipo de esforço $\left(\mathrm{F}_{20,2}=10,89 ; p<\right.$ 0,001 ; poder $=0,99$ ). Os valores dos tempos de esforço (média $\pm \mathrm{ep}$ ), segundo fase competitiva e round são apresentados na Tabela 1 . Nela, contata-se maior tempo de observação no primeiro round da fase eliminatória, e nos rounds de 1 a 3 da fase final. Adicionalmente, aos se compararem os roundsde uma mesma fase competitiva, encontrou-se maior tempo de observação no round 2 comparado aos rounds 1 e 3 na fase eliminatória. Pontua-se, adicionalmente, menor tempo de interação na fase final nos rounds 2 e 3 (Tabela 1).

\section{Discussão}

O propósito do presente estudo foi verificar se há diferenças nos padrões temporais de combates de Muay-Thai ao se considerarem fases competitivas distintas. Destacam-se como principais achados: (i) combates de fases eliminatórias exibem tempo de interação superior aos de fases finais, (ii) no primeiro round o tempo destinado à observação foi superior aos demais tipos de esforço em ambas as fases competitivas, e (iii) o tempo de observação foi maior na fase final quando comparado à fase eliminatória. Ao se agruparem os tempos de observação e preparação como baixa intensidade (BI), e de interação como alta intensidade (AI), a relação AI:BI é de 1:2 em eliminatórias e 1:3 em lutas finais. 
Tabela 1. Dados referentes aos três níveis de esforço em lutas eliminatórias e finais de campeonato nacional de Muay-Thai.

\begin{tabular}{|c|c|c|c|c|c|}
\hline \multirow{2}{*}{ Fase } & \multirow{2}{*}{ Round } & \multirow{2}{*}{ Esforço } & \multirow{2}{*}{ Média \pm ep } & \multicolumn{2}{|c|}{ IC 95\% } \\
\hline & & & & LI & $\mathbf{L S}$ \\
\hline \multirow{12}{*}{ Eliminatória } & \multirow{3}{*}{1} & Observação & $5,9 \pm 0,3$ & 5,3 & 6,6 \\
\hline & & Preparação & $3,2 \pm 0,3 *$ & 2,6 & 3,9 \\
\hline & & Interação & $5,4 \pm 0,3^{*}$ & 4,8 & 6,0 \\
\hline & \multirow{3}{*}{2} & Observação & $8,4 \pm 0,3 \$$ & 7,6 & 9,2 \\
\hline & & Preparação & $2,9 \pm 0,3$ & 2,1 & 3,7 \\
\hline & & Interação & $5,6 \pm 0,3 \#$ & 5,1 & 6,3 \\
\hline & \multirow{3}{*}{3} & Observação & $6,8 \pm 0,4$ & 6,0 & 7,5 \\
\hline & & Preparação & $2,7 \pm 0,4$ & 1,9 & 3,5 \\
\hline & & Interação & $5,5 \pm 0,3 \#$ & 4,8 & 6,3 \\
\hline & \multirow{3}{*}{ Média } & Observação & $7,0 \pm 0,3$ & 6,3 & 7,7 \\
\hline & & Preparação & $2,9 \pm 0,3$ & 2,2 & 3,7 \\
\hline & & Interação & $5,5 \pm 0,3 \dagger$ & 4,9 & 6,2 \\
\hline \multirow{15}{*}{ Final } & \multirow{3}{*}{1} & Observação & $8,9 \pm 0,6 \#$ & 7,7 & 10,2 \\
\hline & & Preparação & $3,6 \pm 0,5^{*}$ & 2,5 & 4,6 \\
\hline & & Interação & $4,6 \pm 0,4^{*}$ & 3,6 & 5,6 \\
\hline & \multirow{3}{*}{2} & Observação & $8,9 \pm 0,6$ & 7,5 & 10,3 \\
\hline & & Preparação & $3,1 \pm 0,5^{*}$ & 2,0 & 4,2 \\
\hline & & Interação & $4,1 \pm 0,5^{*}$ & 3,1 & 4,9 \\
\hline & \multirow{3}{*}{3} & Observação & $6,7 \pm 0,7$ & 5,3 & 8,1 \\
\hline & & Preparação & $2,8 \pm 0,5^{*}$ & 1,7 & 3,9 \\
\hline & & Interação & $3,7 \pm 0,5^{*}$ & 2,6 & 4,7 \\
\hline & \multirow{3}{*}{4} & Observação & $5,8 \pm 0,9$ & 3,9 & 7,8 \\
\hline & & Preparação & $3,0 \pm 0,7$ & 1,6 & 4,4 \\
\hline & & Interação & $2,7 \pm 0,6$ & 1,4 & 4,1 \\
\hline & \multirow{3}{*}{ Média } & Observação & $7,5 \pm 0,7$ & 6,1 & 9,1 \\
\hline & & Preparação & $3,1 \pm 0,5$ & 1,9 & 4,2 \\
\hline & & Interação & $3,7 \pm 0,5$ & 2,6 & 4,8 \\
\hline
\end{tabular}

ep = erro padrão. IC = intervalo de confiança. LI e LS = limites inferior e superior, respectivamente. ${ }^{*}$ Estatisticamente diferente do tempo de observação no mesmo round. \# Estatisticamente diferente do mesmo tipo de esforço e round, segundo fase competitiva. \$ Estatisticamente diferente dos rounds 1 e 3 , para o mesmo tipo de esforço e fase. $†$ Estatisticamente diferente da fase final $(p<0,05)$.

Estudo prévio, com análise de combates de Muay-Thai de nível amador do Uruguai, encontrou tempos de observação, preparação e interação de 5, 2,5 e 5 s, respectivamente (Silva, Del Vecchio, Picanço, Takito, \& Franchini 2011). Embora os valores sejam próximos aos encontrados no presente estudo, os combates de nível nacional brasileiro exibiram de 7 a $8 \mathrm{~s}$ de observação, aproximadamente $3 \mathrm{~s}$ de preparação e 4 a 5,5 s de interação. Neste sentido, a maior diferença se localizou no tempo de observação, provavelmente devido ao maior nível competitivo e ritmo de luta apresentado por lutadores mais experientes. Com efeito, tais achados refutam sugestão prévia de estímulos condicionantes de 2 min, com alternâncias de $5 \mathrm{~s}$ de esforço por $5 \mathrm{~s}$ de pausa no MuayThai (Turner, 2009)

Os valores encontrados no presente estudo na relação AI:BI são ligeiramente diferentes dos observados com lutas uruguaias, da ordem de 2:3 (Silva, Del Vecchio, Picanço, Takito, \& Franchini 2011). De modo amplo, podem-se citar dados referentes ao BJJ, que apresenta relação AI:BI de 1:8 até 1:13 (Andreato, et al., 2015), kickboxing, com 1:1 (Ouergui, et al., 2014), MMA, de 1:2 até 1:4 (Del Vecchio, Hirata, \& Franchini, 2011) e no taekwondo, com AI:BI de 1:7 (Campos, Bertuzzi, Dourado, Santos, \& Franchini, 2012). Por outro lado, todas as modalidades citadas se diferem no tempo total de luta (Muay-Thai $=3$ rounds de $3 \mathrm{~min}$; BJJ= combates de 5 a 10 minutos; kickboxing= 3 a 12 rounds de 2 a 5 minutos; MMA $=3$ a 5 rounds de 5 minutos e taekwondo $=3$ rounds de 2 min). Com relação ao período de intervalo, Muay-Thai, MMA e taekwondo possuem 1 minuto entre rounds, o kickboxing varia de 1 a 2 minutos, enquanto o BJJ não possui período de intervalo, e tais singularidades podem explicar as diferenças na razão AI:BI nestas modalidades.

Nas fases eliminatória e final, constatou-se tempo superior destinado à observação no round 1. Isso poderia ser explicado pelo padrão de atividade dos lutadores durante os momentos 
iniciais da luta, dado que eles optam por observar as tendências de ações do adversário e conhecer suas características de combate, para que nos próximos rounds imponha seu estilo de luta, sem que sejam surpreendidos pelo oponente (Ouergui, et al., 2014). Complementarmente, constata-se menor tempo de interação nas lutas finais, especialmente nos rounds 2 e 3 . É constatado que em combates de Muay-Thai, vencedores tiveram maior eficacia nos golpes deferidos em comparação aos perdedores, ainda no mesmo estudo, foi observado valores de lactato acima do Limiar Anaeróbio $(9,72 \pm 0,6 \mathrm{mmol}$, com valor máximo de $12,55 \pm 1,1 \mathrm{mmol})$, o que indica elevada contribuição do metabolismo anaeróbio glicolítico para suprimento energético (Cappai, et al., 2012; Turner, 2009) especialmente no primeiro round (Crisafulli, et al., 2009). Supõe-se que os atletas que chegaram às finais poderiam estar com grau superior de fadiga em relação às lutas anteriores (Barbas, et al., 2011), fazendo com que os mesmos executem menos períodos de alta intensidade, gerando menor tempo de interação durante as lutas finais. Em lutas sucessivas de BJJ e luta olímpica, observou-se diminuição da força isométrica de preensão manual e da potência de MMII ao longo de combates sucessivos (Andreato, et al., 2015; Barbas, et al., 2011), ao passo que na luta olímpica não foram encontradas mudanças na concentração de cortisol e adrenalina após cinco combates (Fry, Schilling, Fleck, \& Kraemer, 2011).

Por fim, ressalta-se a relevância da utilização de protocolos de treinos baseados nas características temporais de eventos competitivos (Del Vecchio, Hirata, \& Franchini, 2011), fazendo com que os estímulos exibam temporalidade e intensidade próximas das exigências de um combate real (Bridge, McNaughton, Close, \& Drust, 2013), portanto os resultados encontrados no presente estudo podem contribuir no conhecimento do padrão de luta ao longo de uma competição de nível nacional e na organização de meios de treino específico para lutadores de Muay Thai, utilizando a temporalidade encontrada em combates reais.

\section{Conclusão}

Conclui-se que o Muay-Thai se configura como modalidade de combate intermitente, cuja temporalidade é descrita por valores médios na ordem de 7,25 $\pm 0,29 \mathrm{~s}$ de observação, 3,1 $\pm 0,06 \mathrm{~s}$ de Preparação e $5 \pm 0,13 \mathrm{~s}$ de Interação. A relação AI:BI observada foi de 1:2 em combates eliminatórios a 1:3 em lutas de fases finais. Aponta-se que o tempo de interação é inferior em confrontos finais quando comparados aos eliminatórios. Como aplicações práticas, sugerem-se exercícios especiais, como 3 a 5 rounds de 2 minutos de duração, com 1 minuto de intervalo, com sequências de estímulos de Observação, que envolvam deslocamentos e esquivas, com 7 a $8 \mathrm{~s}$, esforços de Preparação, com esquivas e golpes únicos, de 3 a 4 s, e atividades de Interação, com golpes múltiplos e esquivas em alta intensidade, com duração entre 5 e 8 s, perfazendo blocos de 15 a $20 \mathrm{~s}$, os quais se repetem por 8 a 6 vezes em um único round. Isso pode se constituir como estratégia relevante na preparação física de competidores de Muay-Thai.

\section{Referências}

Andreato, L. V., Julio, U. F., Panissa, V. L. G., Esteves, J. V. C., Hardt, F., Moraes, S. M. F., Souza, C. O, \& Franchini, E. (2015). Brazilian jiu-jitsu simulated competition Part II: Physical performance, time-motion, technical-tactical analyses and perceptual responses. Journal of Strength and Conditioning Research, Ahead of Print.

Arriaza, R. (2009). Karate. In R. Kordi, N. Maffulli, R. R. Wroble, \& W. A.Wallace (Eds), Combat Sports Medicine (pp. 287-298). London: Springer-Verlag.

Barbas, I., Fatouros, I. G., Douroudos, I. I., Chatzinikolaou, A., Michailidis, Y., Draganidis, D., Jamurtas, A. Z., Nikolaidis, M. G., Parotsidis, C., Theodorou, A. A., Katrabasas, I., Margonis, K., Papassotiriou, I., \& Taxildaris, K. (2011). Physiological and performance adaptations of elite Greco-Roman wrestlers during a one-day tournament. European journal of applied physiology, 111(7), 1421-1436.

Bridge, C. A., McNaughton, L. R., Close, G. L., \& Drust, B. (2013). Taekwondo exercise protocols do not recreate the physiological responses of championship combat. International Journal of Sports Medicine, 34(7), 573-581. 
Buse, G. J. (2009). Kickboxing. In R. Kordi, N. Maffulli, R. R. Wroble, \& W. A.Wallace (Eds), Combat Sports Medicine (pp. 331-350). London: Springer-Verlag.

Campos, F. A. D., Bertuzzi, R., Dourado, A. C., Santos, V. G. F., \& Franchini, E. (2012). Energy demands in taekwondo athletes during combat simulation. European Journal of Applied Physiology, 112(4), 1221-1228.

Cappai, I., Pierantozzi, E., Tam, E., Tocco, F., Angius, L., Milia, R., Squatrito, S., Concu, A., \& Crisafulli, A. (2012). Physiological responses and match analysis of Muay Thai fighting. International Journal of Performance Analysis in Sport, 12(3), 507-516.

Chaabène, H., Franchini, E., Miarka, B., Selmi, M. A., Mkaouer, B., \& Chamari, K. (2014). Time-Motion Analysis and Physiological Responses to Karate Official Combat Sessions: Is There a Difference Between Winners and Defeated Karatekas? International Journal of Sports Physiology and Performance, 9(2), 302 -308.

Crisafulli, A., Vitelli, S., Cappai, I., Milia, R., Tocco, F., Melis, F., \& Concu, A. (2009). Physiological responses and energy cost during a simulation of a Muay Thai boxing match. Applied Physiology, Nutrition, and Metabolism, 34(2), 143-150.

Del Vecchio, F. B., Bianchi, S., Hirata, S. M., \& Chacon-Mikahil, M. P. T. (2007). Análise morfofuncional de praticantes de brazilian jiu-jitsu e estudo da temporalidade e da quantificação das ações motoras na modalidade. Movimento \& Percepção, 7(10), 263-281.

Del Vechhio, F. B., Hirata, S. M., \& Franchini, E. (2011). A review of time-motion analysis and combat development in Mixed Martial Arts matches at regional level tournaments. Perceptual and Motor Skills, 112(2), 639-648.

Franchini E., \& Del Vechhio, F. B. (2011). Estudos em modalidades esportivas de combate: estado da arte. Revista Brasileira de Educação Física e Esporte, 25(N.esp), 67-81.

Fry, A. C., Schilling, B. K., Fleck, S. J., \& Kraemer, W. J. (2011). Relationships between competitive wrestling success and neuroendocrine responses. Journal of Strength and Conditioning Research, 25(1), 40-45.

Heller, J., Peric, T., Dlouhá, R., Kohliková, E., Melichna, J., \& Nováková, H. (1998). Physiological profiles of male and female taekwon-do (ITF) black belts. Journal of Sports Science, 16(3), 243-249.

Marcon, G., Franchini, E., Jardim, J. R., Barros Neto, T. L. (2010). Structural analysis of action and time in sports: judo. Journal of Quantitative Analysis in Sport, 6(4), 1-15.

Matsushigue, K. A., Hartmann, K., \& Franchini, E. (2009). Taekwondo: Physiological responses and match analysis. Journal of Strength and Conditioning Research, 23(4), 1112-1117.

Miarka, B., Hayashuda, C. R., Julio, U.F., Calmet, M., \& Franchini, E. (2011). Objectivity of FRAMISoftware for Judo Match Analysis. International Journal of Performance Analysis in Sport, 11(2), 254-266.

Miarka, B., Panissa, V., Julio, U. F.,Vecchio, F. B., Calmet, M., \& Franchini E. (2012). A comparison of time-motion performance between age groups in judo matches. Journal of Sports Sciences, 30(9), 899-905.

Ouergui, I., Hssin, N., Haddad, M., Franchini, E., Behm, D., Wong, P., Gmada, N., \& Bouhlel, E. (2014). Time motion analysis of international kickboxing competition. Journal of Strength and Conditioning Research, 28(12), 3537-3543.

Silva, J. J. R., Del Vecchio, F. B., Picanço, L. M., Takito, M. Y., \& Franchini, E. (2011). Time-Motion analysis in Muay-Thai and Kick-Boxing amateur matches. Journal of Human Sport \& Exercise, 6(3), 1-7.

Turner, A. N. (2009). Strength and Conditioning for Muay Thai Athletes. Strength and Conditioning Journal, 31(6), 78-92.

Ugras, A. F. (2013). Effect of high intensity interval training on elite athletes' antioxidant status. Science \& Sports, 28(5), 253-259.

\section{Author's biographical data}

Fabrício Boscolo Del Vecchio: Doutor em Educação Física pela FEF/UNICAMP, orientador de doutorado na ESEF/UFPel. Líder do grupo de estudos e pesquisas em treinamento esportivo e desempenho físico da ESEF/UFPel. Faixa preta de judô 2 o dan. E-mail: fabricio boscolo@uol.com.br. 
Jerônimo Jaspe Rodrigues Silva: Bacharel em Educação Física pela ESEF/UFPel. Mestrando em Educação Física na ESEF/UFRGS. Tem experiência com preparação física de lutadores e foi competidor de Muay-Thai. E-mail: jeronimojrsilva@gmail.com.

Charles Bartel Farias: Cursa Bacharelado em Educação Física na ESEF/UFPel. Tem experiência com preparação física de lutadores e é competidor de Muay-Thai. Membro do grupo de estudos e pesquisas em treinamento esportivo e desempenho físico da ESEF/UFPel. E-mail: charlesbartelcrn@gmail.com. 